\title{
Far-UV spectroscopy of two extremely hot, helium-rich white dwarfs ${ }^{\star}$
}

\author{
K. Werner ${ }^{1}$, T. Rauch ${ }^{1}$, and J. W. Kruk ${ }^{2}$ \\ ${ }^{1}$ Institute for Astronomy and Astrophysics, Kepler Center for Astro and Particle Physics, Eberhard Karls University, Sand 1, \\ 72076 Tübingen, Germany \\ e-mail: werner@astro.uni-tuebingen.de \\ 2 NASA Goddard Space Flight Center, Greenbelt, MD 20771, USA
}

Received 16 December 2016 / Accepted 23 February 2017

\begin{abstract}
A large proportion of hot post-asymptotic giant branch stars and white dwarfs (WDs) are hydrogen-deficient. Two distinct evolutionary sequences have been identified. One of them comprises stars of spectral type [WC] and PG1159, and it originates from a late heliumshell flash, creating helium-rich stellar atmospheres with significant admixtures of carbon (up to about 50\%, mass fraction). The other sequence comprises stars of spectral type $\mathrm{O}(\mathrm{He})$ and luminous subdwarf $\mathrm{O}$ stars which possibly are descendants of $\mathrm{RCrB}$ stars and extreme helium stars. Their carbon abundances are significantly lower (of the order of $1 \%$ or less) and it is thought that they originate from binary-star evolution (through merger or common-envelope evolution). Here we investigate two of the three hottest known helium-rich (DO) WDs (PG 1034+001 and PG 0038+199). They are the only ones for which spectra were recorded with the Far Ultraviolet Spectroscopic Explorer and the Hubble Space Telescope, allowing a comprehensive ultraviolet spectral analysis. We find effective temperatures of $T_{\text {eff }}=115000 \pm 5000 \mathrm{~K}$ and $125000 \pm 5000 \mathrm{~K}$, respectively, and a surface gravity of $\log g=7 \pm 0.5$. In both stars, nitrogen is strongly oversolar while $\mathrm{C}$ and $\mathrm{O}$ are significantly subsolar. For all other assessed metals $(\mathrm{Ne}, \mathrm{Si}, \mathrm{P}, \mathrm{S}, \mathrm{Ar}, \mathrm{Fe}$, and $\mathrm{Ni}$ ) we find abundances close to solar. We conclude that these WDs are immediate descendants of $\mathrm{O}(\mathrm{He})$ stars and, hence, result from close-binary evolution.
\end{abstract}

Key words. stars: abundances - stars: atmospheres - stars: evolution - stars: AGB and post-AGB - (stars:) white dwarfs

\section{Introduction}

About a quarter of all post-asymptotic giant branch (post-AGB) stars are hydrogen-deficient. According to the carbon abundance in the helium-dominated atmospheres, they fall into two distinct groups that probably arise from different evolutionary scenarios. The first group, comprising [WC] central stars of planetary nebulae and PG1159 stars, has high C mass fractions (up to 50\%). Their H-deficiency was caused by a late helium-shell flash (late thermal pulse, LTP; see, e.g., the review by Werner \& Herwig 2006). The other group, comprising RCrB stars, extreme helium (EHe) stars, luminous helium-subdwarf $\mathrm{O}$ stars, and their hottest members, the $\mathrm{O}(\mathrm{He})$ stars, have lower $\mathrm{C}$ abundances (of the order of $1 \%$ or less). Their origin is debated, but there is evidence that they stem from close-binary evolution. They could be the result of binary white dwarf (WD) mergers (or an AGB-star and WD merger), or the result of envelope stripping by commonenvelope evolution (see, e.g., discussion in Reindl et al. 2014b). The further fate of these stars depends on the retained hydrogen mass. Without any hydrogen left, the stars will become heliumrich WDs (non-DAs), or H-rich (DA) WDs otherwise.

In this paper, we focus on two extremely hot helium-rich WDs (spectral type DO) which are located at the hot end of the WD cooling sequence in the Hertzsprung-Russell diagram and, hence, could be immediate successors of the $\mathrm{O}(\mathrm{He})$ stars.

\footnotetext{
* Based on observations made with the NASA-CNES-CSA Far Ultraviolet Spectroscopic Explorer.
}

We present a spectral analysis to check for this evolutionary connection.

Both stars analyzed in this paper were discovered in the Palomar-Green Survey (Green et al. 1986) and were previously subject to spectral analyses. What is new in our work is the analysis of far-ultraviolet spectra taken with the Far Ultraviolet Spectroscopic Explorer (FUSE). This enables unique access to element abundances and an effective temperature determination with unprecedentedly high precision.

In the following, we introduce our program stars (Sect. 2) and present the utilized ultraviolet (UV) observations and spectral line identifications (Sect.3). After an overview of the employed model atmospheres and atomic data (Sect. 4), we present our results (Sect. 5). They supersede preliminary results from ongoing work published in Mahsereci (2011) and Mahsereci et al. (2012). We conclude with a summary and discussion (Sect. 6).

\section{The program stars}

About seventy DO WDs are known (Werner et al. 2014; Reindl et al.2014a). They define the hot end of the WD cooling sequence of non-DA WDs. Their effective temperatures are in the range $T_{\text {eff }} \approx 50000-120000 \mathrm{~K}$. At lower temperatures the Herich WDs are, by definition, of spectral type DB, being too cool to exhibit ionized helium lines. PG 1034+001 and PG 0038+199 belong to the subgroup of "hot DOs", defined by the absence of neutral helium lines $\left(T_{\text {eff }} \gtrsim 70000 \mathrm{~K}\right)$. In fact, our analysis reveals that they are slightly hotter than previously thought 
$\left(T_{\text {eff }}=115000 \pm 5000 \mathrm{~K}\right.$ and $125000 \pm 5000 \mathrm{~K}$, respectively) so that they are among the three hottest known DOs, joined by SDSS J151215.72+065156.3 $(120000 \pm 15000 \mathrm{~K})$. We note that the hottest known He-dominated object KPD 0005+5106 was reclassified from $\mathrm{DO}$ to $\mathrm{O}(\mathrm{He})$ because of its relatively low gravity $\left(T_{\text {eff }}=195000 \pm 15000 \mathrm{~K}, \log g=6.7 \pm 0.3\right.$, Werner \& Rauch 2015).

\section{1. $P G 1034+001$}

A detailed analysis with non-local thermodynamic equilibrium (non-LTE) model atmospheres was presented by Werner et al. (1995) using UV spectra recorded with the Hubble Space Telescope (HST) and optical spectra. They derived $T_{\text {eff }}=$ $100000_{-10000}^{+15000} \mathrm{~K}$ and $\log g=7.5 \pm 0.3$. They also measured the abundances for $\mathrm{C}, \mathrm{N}, \mathrm{O}, \mathrm{Si}, \mathrm{Fe}, \mathrm{Ni}$, and, later of $\mathrm{Ar}$ (Werner et al. 2007) from FUSE data. The main uncertainty, however, was the lack of an ionization balance that would allow us to put stringent error limits for the effective temperature. The later detection of Fe VIII lines in FUSE spectra suggested a significantly higher temperature $\left(T_{\mathrm{eff}} \approx 120000 \mathrm{~K}\right.$, Werner et al. 2011$)$, so that a reassessment of all element abundances is appropriate. A search for the $\mathrm{H} \alpha$ line (Werner 1996) yielded an upper limit for the hydrogen abundance of $\mathrm{H}<0.012$ (mass fraction).

\section{2. $P G 0038+199$}

From optical spectroscopy, $T_{\text {eff }}=115000 \pm 11500 \mathrm{~K}$ and $\log g=$ $7.5 \pm 0.3$ were derived (Dreizler \& Werner 1996). From the presence of an $\mathrm{N} \mathrm{V}$ emission line, an abundance of $\mathrm{N}=3.5 \times 10^{-3}$ was estimated. Like in the case of PG 1034+001, the detection of Fe VIII lines hinted at a higher temperature ( $\approx 140000 \mathrm{~K})$. Interestingly, PG 0038+199 is the only DO besides HD 149499B $\left(T_{\text {eff }}=49500 \pm 2500 \mathrm{~K}\right.$, Werner et al. 1995) in which hydrogen was found (i.e., they are the only representatives of the spectral class DOA). From the detected $\mathrm{H} \alpha$ emission core, Dreizler et al. (1997) derived H $=0.05$. UV spectra of PG 0038+199 were not yet investigated.

\section{Observations and line identifications}

PG0038+199 and PG 1034+001 were observed with FUSE (912-1180 $\AA$; resolving power $R \approx 20000$; spectra displayed in Figs. 1 and 2) in 2000 and 2004, respectively. The data reduction warrants detailed description (see below), because our analysis is mainly based on it. We also use spectra of PG 1034+001 taken with the GHRS instrument on HST in 1992, covering five about $35 \AA$ wide wavelength intervals (grating G160M, $R \approx 9000$; displayed in Fig. 3). They were first analyzed by Werner et al. (1995) and in the present study we draw on them for consistency checks of our results obtained from the FUSE data. More recently (2010 and 2011), both stars were observed with the STIS instrument aboard HST (1192-1245 A; grating G140M, $R \approx 12000$; displayed in the top panel of Fig. 3 and in Fig. 4).

The raw FUSE data for the program stars were retrieved from the Mikulski Archive for Space Telescopes (MAST). The observation IDs were P1042003 for PG 1034+001, and P1040201, M1141101 for PG 0038+199. PG 1034+001 was observed through the 4 " MDRS aperture, PG0038+199 was observed through the $30^{\prime \prime}$ LWRS spectrograph aperture, and all the data were obtained in time-tag mode.

Misalignment of the channels for the MDRS observation P1042003 caused some loss of flux. The LiF2 exposures were photometric in 11 of the 13 exposures; the remaining two exposures suffered flux losses of $\approx 30 \%$ due to the star being on the edge of the slit. The LiF1 exposures were similar, though with losses of a few percent in about half of the 11 good exposures. The fluxes in the non-photometric exposures were normalized to the good exposures. None of the exposures in the $\mathrm{SiC}$ channels were clearly photometric. The exposures with less than $40 \%$ of the peak flux were discarded, and the rest were normalized to match the LiF spectra in the regions of overlap. Exposure 14 was lost in all channels due to the detector high voltage being off. The effective exposure times ranged from a low of $2.3 \mathrm{ksec}$ in $\mathrm{SiC} 2 \mathrm{a}$ to $5.9 \mathrm{ksec}$ in LiF1a.

The P1040201 observation of PG 0038+199 was photometric in all channels for all exposures, with sub-percent variations in flux from one exposure to the next. The M1141101 observation was performed as a channel-alignment activity, with the star being scanned across the slit in both axes. The spectra were normalized to match the P1040201 data to account for time when the star was outside of the slits. The CalFUSE pipeline properly accounted for the changing wavelength zeropoint during each exposure: the spectral resolution of the M1141101 data appeared identical to that of the P1040201 data. The effective exposure time varied only slightly, from a low of $17.7 \mathrm{ksec}$ in $\mathrm{SiC} 1$ to $17.9 \mathrm{ksec}$ in the other channels.

Raw data were processed twice with CalFUSE v3.2.3: once with screening parameters set to extract data only during orbital Night, and once to extract data during orbital Day. Zeropoint offsets in the wavelength scale were adjusted for each exposure by shifting each spectrum to coalign narrow interstellar absorption features. The individual exposures from each observation were then combined to form composite Day and Night spectra for each channel. The Day and Night spectra were then compared at the locations of all the known airglow emission lines. If the Day spectra showed any excess flux in comparison to the Night spectra at those wavelengths, the corresponding pixels in the Day spectra were flagged as bad and were not included in subsequent processing. There was no significant airglow emission in the MDRS PG 1034+001 Night spectra. For the LWRS PG 0038+199 observations, significant airglow was present during orbital Night only at Lyman $\beta$, and faint emission can be discerned at Lyman $\gamma$. This residual emission would affect fits to the interstellar absorption at these wavelengths, but has no impact on any of the analyses we present. The Day airglow emission in the PG 0038+199 spectra was noteworthy in that weak He I $584 \AA$ emission was visible in second order, appearing at $1168 \AA$. This emission would fill in the C IV photospheric absorption feature at that wavelength if the orbital Day data had not been excluded.

The LWRS LiF1b spectra had to be corrected as usual for the shadow cast by the detector repeller grid wires (the so-called "worms" - see Sect. 7.3.2 of the FUSE Data Handbook ${ }^{1}$ ). This is done by computing a highly-smoothed ratio of the observed $\mathrm{LiF} 2 \mathrm{a}$ and LiF1b spectra, and re-normalizing the LiF1b spectrum by this ratio. The continuum shape at these wavelengths is thus derived solely from the LiF2a spectrum, but both spectra contribute to the information on scales narrower than 100 pixels. The grid-wire shadows are in different locations for the MDRS spectra. The same correction procedure was applied to the SiC1b and LiF2b PG 1034+001 spectra, using SiC2a and LiF1a as the continuum template. A similar correction was also required for LiF1b at wavelengths longward of $1175 \AA$; in this case the continuum template was derived by extrapolating a power-law fit to the spectrum at shorter wavelengths.

http://archive.stsci.edu/fuse/dh.html 
K. Werner et al.: FUV spectroscopy of two extremely hot, helium-rich white dwarfs

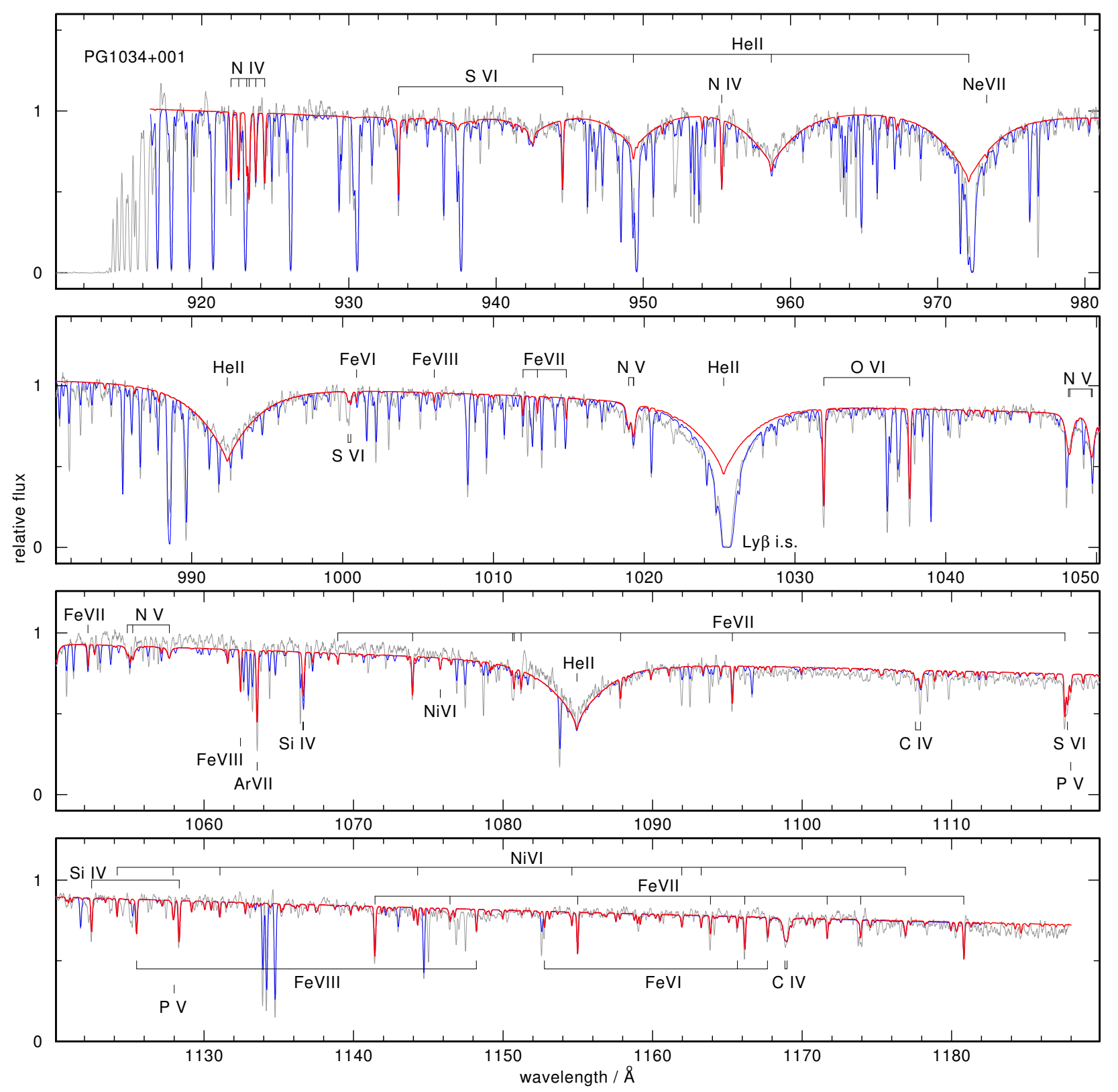

Fig. 1. FUSE spectrum of PG $1034+001$ (thin black line) compared to a photospheric model spectrum (thick red line) with the finally adopted parameters as listed in Table 1. Overplotted in blue is the same model including ISM lines. Prominent spectral lines are identified.

The final step was to combine the spectra from the four instrument channels into a single composite spectrum. Because of residual distortions in the wavelength scale in each channel, additional shifts of localized regions of each spectrum were required to coalign the spectra; these shifts were typically only one to two pixels. Bad pixels resulting from detector defects were flagged at this point and excluded from further processing. Finally, the spectra were resampled onto a common wavelength scale and combined, weighting by the signal-to-noise ratio $(\mathrm{S} / \mathrm{N})$ on a pixel-by-pixel basis. The wavelength zeropoint was set by placing the interstellar medium (ISM) lines at a heliocentric velocity of $-27 \mathrm{~km} \mathrm{~s}^{-1}$ for PG $1034+001$ (Werner et al. 1995) and $-5.1 \mathrm{~km} \mathrm{~s}^{-1}$ for PG 0038+199 (Williger et al. 2005).

The final spectrum has $S / N=18-47$ per $0.013 \AA$ pixel in the continuum for PG 1034+001, and 26-47 per pixel for PG 0038+199. The effects of fixed-pattern noise are minimized by the fact that the positions of the spectra on the detectors varied during each observation, and by the fact that nearly every wavelength bin was sampled by at least two different detectors.

The FUSE spectra of both stars have a rather limited amount of photospheric lines (Tables 2 and 3). Most of them stem from highly ionized iron (Fe VI-VIII). We also see a few lines of C IV, N IV-V, O VI, Ne VII, Si IV-V, P V, S VI, Ar VII, and Ni VI. Not all of the ions are seen in both stars; a detailed discussion follows in the description of the spectral analysis results. All spectra shown in this paper were wavelength shifted such that the photospheric lines are at rest wavelengths. The FUSE spectrum of PG 0038+199 is strongly contaminated by interstellar absorption lines, while that of PG $1034+001$ is less affected.

To identify lines from the ISM and to judge their potential contamination of photospheric lines, we used the program OWENS (Hébrard et al. 2002; Hébrard \& Moos 2003). This can consider different clouds with individual radial and turbulent velocities, temperatures, column densities, and 

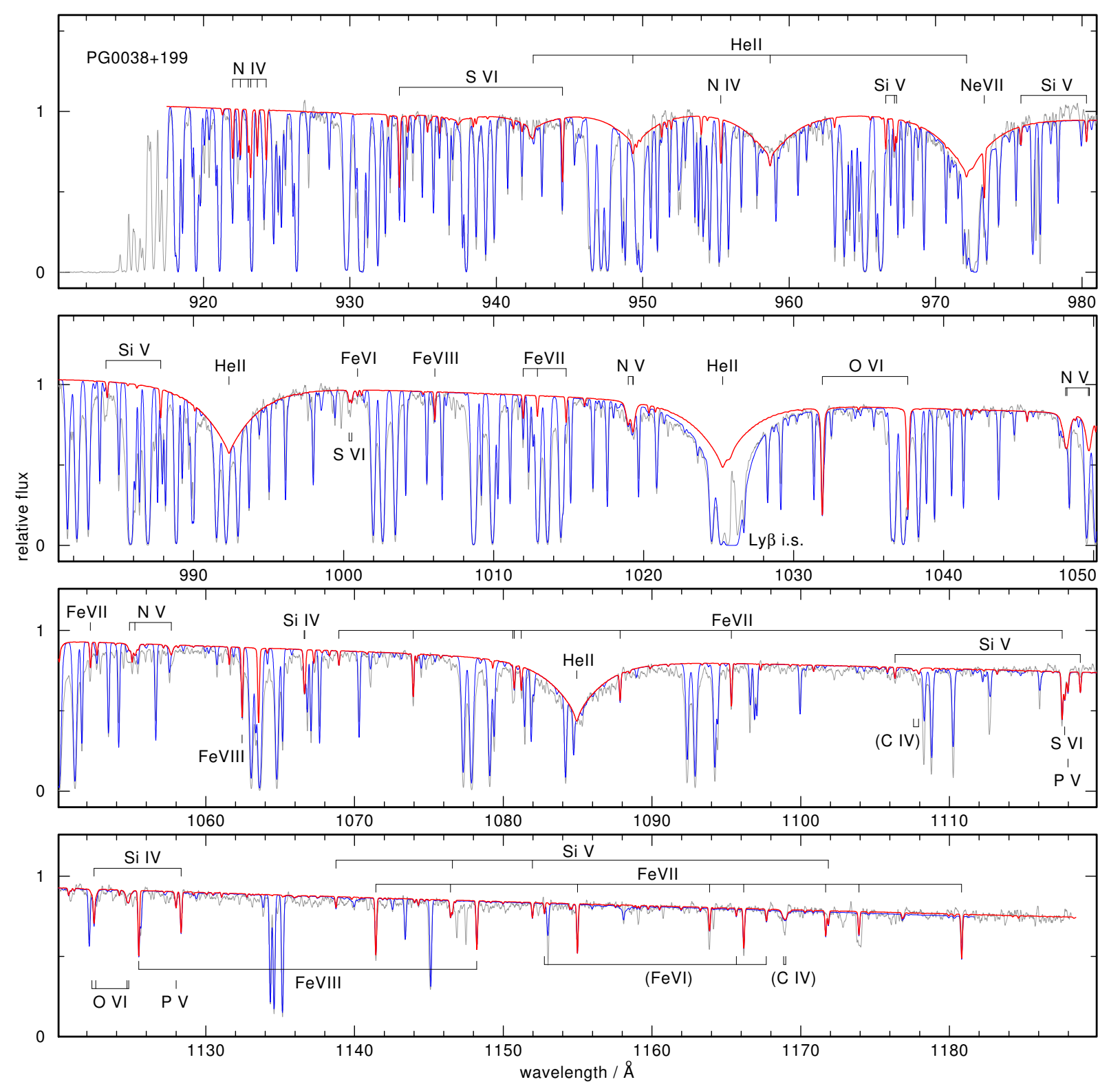

Fig. 2. As Fig. 1, for PG 0038+199. Ion names in brackets denote very weak lines visible in the computed spectrum, but not in the observation.

chemical compositions. In the FUSE and HST observations of PG 1034+001, we identified and modeled ISM lines (one cloud with radial velocity $v_{\text {rad }}=-55 \mathrm{~km} \mathrm{~s}^{-1}$ ) of $\mathrm{HI}, \mathrm{H}_{2}$, D I, C II-III, N I-II, O I, VI, Mg II, Si II, P II, S VI, Ar I, and Fe II, and in the case of PG0038+199, ISM lines (two clouds with $v_{\text {rad }}=-15$ and $+55 \mathrm{~km} \mathrm{~s}^{-1}$ ) of H I, $\mathrm{H}_{2}$, HD, D I, C I-III, N I-II, O I, VI, Si II, P II, S III, Ar I, and Fe II. The blue graphs in Figs. 1-4 show the photospheric models including the ISM lines.

\section{Model atoms and model atmospheres}

We used the Tübingen Model Atmosphere Package (TMAP ${ }^{2}$ ) to compute non-LTE, plane-parallel, line-blanketed atmosphere models in radiative and hydrostatic equilibrium (Werner \& Dreizler 1999; Werner et al. 2003, 2012). Table 4 summarizes the number of considered non-LTE levels and radiative transitions between them. All model atoms were built from the publicly available Tübingen Model Atom Database

\footnotetext{
2 http://astro.uni-tuebingen.de/ TMAP
}

(TMAD), which is comprised of data from different sources, namely Bashkin \& Stoner (1975), the databases of the National Institute of Standards and Technology (NIST ${ }^{3}$ ), the Opacity Project $^{4}$ (OP, Seaton et al. 1994), CHIANTI ${ }^{5}$ (Dere et al. 1997; Landi et al. 2013), as well as the Kentucky Atomic Line List ${ }^{6}$.

For iron and nickel we used a statistical approach, employing typically seven superlevels per ion linked by superlines, together with an opacity sampling method (Anderson 1989; Rauch \& Deetjen 2003). Ionization stages Fe VI-IX and Ni V-VIII augmented by single, ground-level stages Fe $\mathrm{X}$ and Ni IX were considered. We used the complete line list of Kurucz (so-called LIN lists, comprising about $1.2 \times 10^{6}$ and $1.6 \times$ $10^{6}$ lines of the considered ions of $\mathrm{Fe}$ and Ni; Kurucz 1991, 2009, 2011) for the computation of the non-LTE population numbers, and the so-called POS lists (that include only the subset of lines

\footnotetext{
3 http://wWw.nist.gov/pml/data/asd.cfm

4 http://cdsweb.u-strasbg.fr/topbase/topbase.html

5 http://www. chiantidatabase.org

6 http://www.pa.uky. edu/ peter/atomic
} 
K. Werner et al.: FUV spectroscopy of two extremely hot, helium-rich white dwarfs

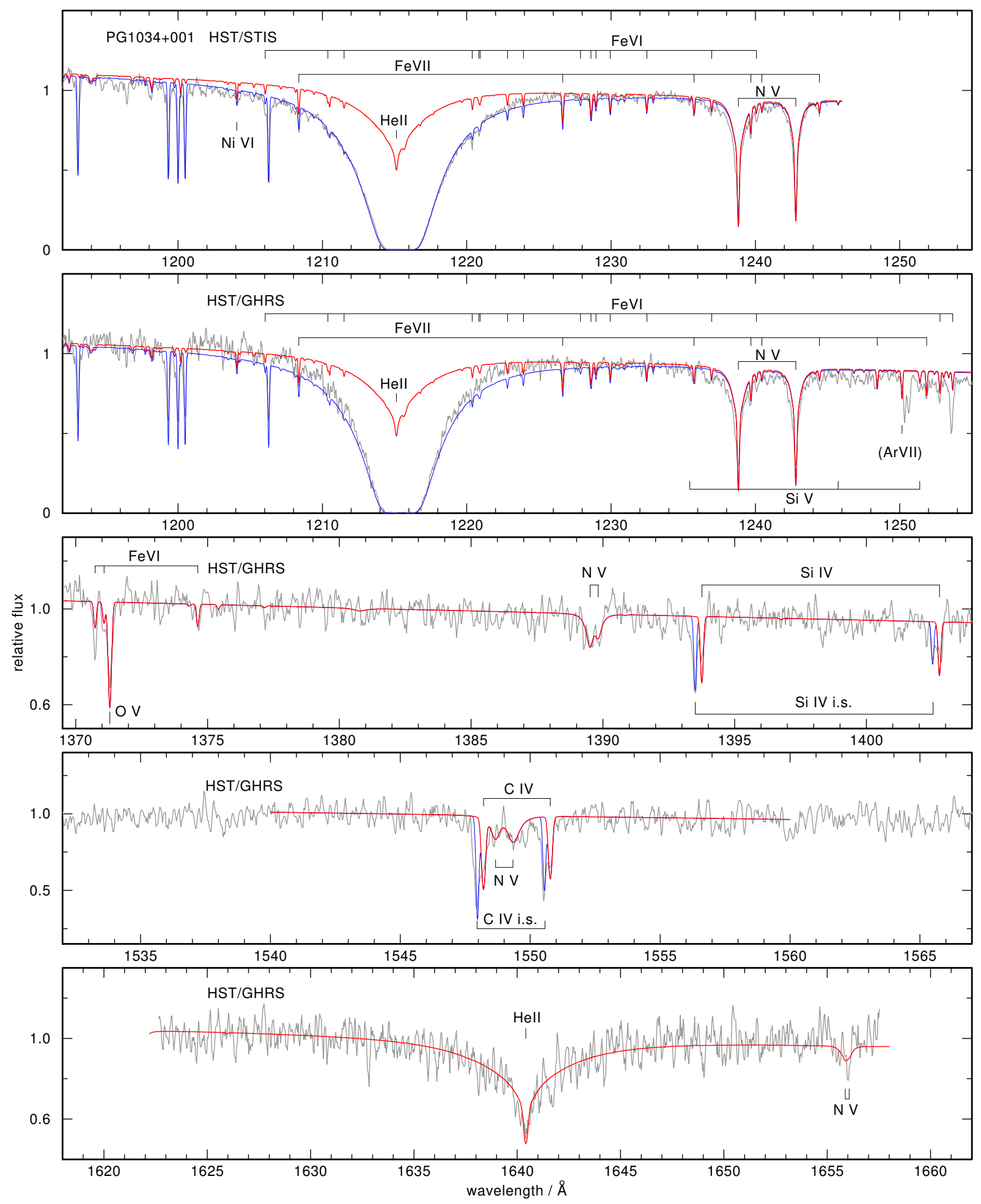

Fig. 3. As Fig. 1, but for the HST spectra of PG 1034+001.

with well known, experimentally observed line positions) for the final spectrum synthesis.

\section{Spectral line fitting procedure and results}

For the spectral analysis we proceeded as follows. First we computed a grid of almost pure helium non-LTE models (with a marginal admixture of $0.25 \%$ hydrogen). Then, for each of the heavier elements detected in the observations $(\mathrm{C}, \mathrm{N}, \mathrm{O}, \mathrm{Ne}, \mathrm{Si}$,
$\mathrm{P}, \mathrm{S}, \mathrm{Ar}, \mathrm{Fe}, \mathrm{Ni}$ ), an independent non-LTE line-formation calculation for atomic populations was performed with fixed model structure. Finally, a spectral synthesis calculation (formal solution) was performed collecting from these separate models the population numbers of all species. These model spectra were used to determine $T_{\text {eff }}, \log g$, and metal abundances from the UV observations.

To check for the influence of metals on the model structure and on the line profiles, we calculated for each star a model with 


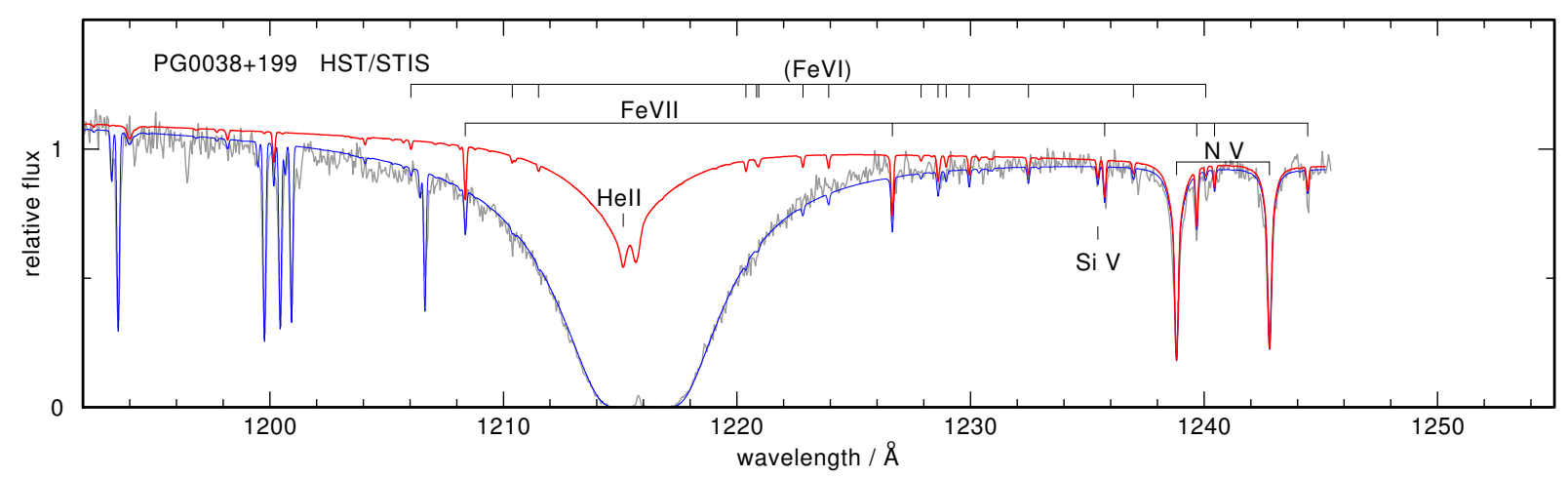

Fig. 4. As Fig. 1, but for the HST spectrum of PG 0038+199.

Table 1. Final adopted parameters for PG 1034+001 and PG 0038+199. The last column shows solar abundance values.

\begin{tabular}{rrrr}
\hline \hline & PG 1034+001 & PG 0038+199 & Sun $^{a}$ \\
\hline$T_{\text {eff }} / \mathrm{K}$ & 115000 & 125000 & \\
$\log g$ & 7.0 & 7.0 & \\
$\mathrm{H}$ & $<0.012$ & 0.02 & 0.74 \\
$\mathrm{He}$ & 0.99 & 0.97 & 0.25 \\
$\mathrm{C}$ & $2.3 \times 10^{-4}$ & $<1.0 \times 10^{-4}$ & $2.4 \times 10^{-3}$ \\
$\mathrm{~N}$ & $7.0 \times 10^{-3}$ & $1.0 \times 10^{-2}$ & $6.9 \times 10^{-4}$ \\
$\mathrm{O}$ & $1.0 \times 10^{-5}$ & $1.0 \times 10^{-4}$ & $5.7 \times 10^{-3}$ \\
$\mathrm{Ne}$ & $<1.3 \times 10^{-3}$ & $<1.3 \times 10^{-3}$ & $1.3 \times 10^{-3}$ \\
$\mathrm{Si}$ & $5.0 \times 10^{-4}$ & $1.0 \times 10^{-3}$ & $6.6 \times 10^{-4}$ \\
$\mathrm{P}$ & $2.9 \times 10^{-6}$ & $1.0 \times 10^{-5}$ & $5.8 \times 10^{-6}$ \\
$\mathrm{~S}$ & $5.0 \times 10^{-5}$ & $1.0 \times 10^{-4}$ & $3.1 \times 10^{-4}$ \\
$\mathrm{Ar}$ & $1.0 \times 10^{-4}$ & - & $7.3 \times 10^{-5}$ \\
$\mathrm{Fe}$ & $1.3 \times 10^{-3}$ & $2.6 \times 10^{-3}$ & $1.3 \times 10^{-3}$ \\
$\mathrm{Ni}$ & $1.0 \times 10^{-4}$ & $<3.0 \times 10^{-4}$ & $7.1 \times 10^{-5}$ \\
\hline
\end{tabular}

Notes. Abundances in mass fractions and surface gravity $g$ in $\mathrm{cm} \mathrm{s}^{-2}$. ${ }^{(a)}$ Solar abundances from Asplund et al. (2009).

parameters as determined above, but now including all considered elements (up to iron). We found that the line profiles in the UV change only marginally and, thus, further fine-tuning of the abundances lead to small corrections, however, with one notable exception. The only detectable neon line (Ne VII $\lambda 973.3 \AA$ ) is much weaker in the metal-line blanketed models and, hence, requires a much higher neon abundance (to be increased by a factor of about 50) to achieve a good model fit to the observation. All computed spectra presented in this paper are from the final metal-line blanketed models.

PG 0038+199 has a significant amount of hydrogen in its atmosphere but it was verified that this does not affect the emergent spectrum compared to a pure-helium model, except of course the $\mathrm{H} \alpha$ profile in the optical. The final models were used to compute optical spectra as well, in order to check their consistency with observations.

Effective temperature and surface gravity. Sensitive temperature indicators are offered by a number of ionization balances. Iron is most useful because it shows lines of three ionization stages (Fe VI-VIII). The temperature can be determined with an accuracy of $5000 \mathrm{~K}$. Too high $T_{\text {eff }}$ causes the Fe VI lines to disappear, while the Fe VIII lines vanish at too low $T_{\text {eff }}$. Additionally, we can utilize the N IV/N V and Si IV/Si V balances. We find
Table 2. Lines of light metals identified in the UV spectra of PG 1034+001 and PG 0038+199.

\begin{tabular}{|c|c|c|c|}
\hline Wavelength & Ion & Transition & $\operatorname{Star}^{a}$ \\
\hline $1107.59,1107.93$ & CIV & $3 p-4 d$ & $\mathrm{a}$ \\
\hline $1168.85,1168.99$ & C IV & $3 d-4 f$ & $\mathrm{a}$ \\
\hline $1550.77,1548.20$ & C IV & $2 s-2 p$ & $\mathrm{a}$ \\
\hline \multicolumn{3}{|l|}{ 921.99, 922.52, 923.06, } & $a, b$ \\
\hline 955.33 & N IV & $2 s 2 p^{1} P^{o}-2 p^{2}{ }^{1} S$ & $\mathrm{a}$ \\
\hline $1018.97,1019.29,1019.31$ & $\mathrm{Nv}$ & $4 p-6 d$ & $a, b$ \\
\hline $1048.13,1048.23$ & $\mathrm{Nv}$ & $4 d-6 f$ & $a, b$ \\
\hline $1049.65,1049.71$ & $\mathrm{NV}$ & $4 f-6 g$ & $a, b$ \\
\hline $1054.87,1055.24,1057.68$ & $\mathrm{NV}$ & $4 p-6 s$ & $a, b$ \\
\hline $1238.82,1242.80$ & $\mathrm{Nv}$ & $2 s-2 p$ & $a, b$ \\
\hline $1389.51,1389.82$ & $\mathrm{NV}$ & $4 s-5 p$ & $\mathrm{a}$ \\
\hline $1548.67,1549.34$ & $\mathrm{Nv}$ & $4 p-5 d$ & a \\
\hline $1655.86,1656.07$ & $\mathrm{~N} \mathrm{v}$ & $4 d-5 p$ & $\mathrm{a}$ \\
\hline 1371.30 & $\mathrm{Ov}$ & $2 s 2 p^{1} P^{o}-2 p^{2}{ }^{1} D$ & $\mathrm{a}$ \\
\hline $1031.91,1037.61$ & O VI & $2 s-2 p$ & $a, b$ \\
\hline $1122.34,1122.60$ & O VI & $4 d-5 f$ & $\mathrm{~b}$ \\
\hline $1124.70,1124.82$ & O VI & $4 f-5 g$ & $\mathrm{~b}$ \\
\hline 973.33 & $\mathrm{Ne}$ VII & $2 \mathrm{~s} 2 \mathrm{p}^{1} \mathrm{P}^{\mathrm{o}}-2 \mathrm{p}^{2}{ }^{1} \mathrm{D}$ & a \\
\hline 1066.61-1066.65 & Si IV & $3 d-4 f$ & $a, b$ \\
\hline $1122.49,1128.34$ & Si IV & $3 p-3 d$ & $a, b$ \\
\hline $1393.75,1402.77$ & Si IV & $3 s-3 p$ & $\mathrm{a}$ \\
\hline 966.599 & Si v & $3 p^{3} D-3 d^{3} F^{o}$ & $\mathrm{~b}$ \\
\hline $975.83,980.31$ & Siv & $3 p^{3} P-3 d^{3} D^{o}$ & $\mathrm{~b}$ \\
\hline 984.17: & Si V & $3 p^{3} D-3 d^{3} P^{o}$ & $\mathrm{~b}$ \\
\hline 987.81: & Siv & $3 p^{1} D-3 d^{1} F^{o}$ & $\mathrm{~b}$ \\
\hline 1106.34, 1118.81, 1138.75, & & & \\
\hline $\begin{array}{l}1146.58,1151.96,1171.85 \\
1235.45\end{array}$ & $\begin{array}{l}\text { Siv } \\
\text { Si V }\end{array}$ & $\begin{array}{l}3 s^{3} P^{0}-3 p p^{3} P \\
3 s^{3} P^{0}-3 p^{3} D\end{array}$ & $\begin{array}{l}\mathrm{b} \\
\mathrm{a}, \mathrm{b}\end{array}$ \\
\hline 1251.39 & Siv & $3 s^{3} P^{o}-3 p^{3} D$ & $\mathrm{a}$ \\
\hline $1117.98,1128.01$ & $\mathrm{PV}$ & $3 s-3 p$ & $a, b$ \\
\hline $933.38,944.52$ & S VI & $3 s-3 p$ & $a, b$ \\
\hline $1000.37,1000.54$ & S VI & $4 d-5 f$ & $a, b$ \\
\hline 1117.76 & S VI & $4 f-5 g$ & $a, b$ \\
\hline 1063.55 & Ar VII & $3 s 3 p^{1} P^{o}-3 p^{2}{ }^{1} D$ & a \\
\hline
\end{tabular}

Notes. Ordered by elements and ionization stages. Wavelengths in $\AA$. No spectra available for PG $0038+199$ at $\lambda>1246 \AA$. Colons denote uncertain identifications of very weak or blended lines. ${ }^{(a)} \mathrm{a}$ and $\mathrm{b}$ denotes visibility in PG 1034+001 and PG 0038+199, respectively.

$T_{\text {eff }}=115000 \pm 5000 \mathrm{~K}$ for PG $1034+001$ and $125000 \pm 5000 \mathrm{~K}$ for PG 0038+199. The He II lines in the UV spectra were used to constrain the surface gravity. We determine log $g=7.0 \pm 0.5$ for both stars. Varying $\log g$ in the models by \pm 0.5 dex slightly 
K. Werner et al.: FUV spectroscopy of two extremely hot, helium-rich white dwarfs

Table 3. Like Table 2, but here for iron and nickel.

\begin{tabular}{|c|c|c|c|}
\hline Wavelength & Ion & Transition & Star \\
\hline 1000.93 & $\mathrm{Fe} \mathrm{VI}$ & $4 s^{2} F-4 p^{2} D^{o}$ & $\mathrm{a}$ \\
\hline 1120.93:, 1121.15: & $\mathrm{Fe}$ VI & $4 s^{2} F-4 p^{2} F^{o}$ & a \\
\hline $1152.77,1167.69$ & $\mathrm{Fe}$ VI & $4 s^{2} G-4 p^{2} F^{o}$ & $\mathrm{a}$ \\
\hline 1165.67 & Fe VI & $4 \mathrm{~s}^{2} \mathrm{P}-4 \mathrm{p}^{2} \mathrm{P}^{\mathrm{o}}$ & $\mathrm{a}$ \\
\hline $\begin{array}{l}1206.04,1211.50,1220.84, \\
1227.88\end{array}$ & $\mathrm{Fe} \mathrm{VI}$ & $4 s^{4} P-4 p^{4} P^{0}$ & $\mathrm{a}$ \\
\hline $1210.38,1220.39$ & $\mathrm{Fe}$ VI & $4 s^{2} \mathrm{P}-4 \mathrm{p}^{2} \mathrm{D}^{\mathrm{o}}$ & $\mathrm{a}$ \\
\hline $\begin{array}{l}1220.93,1222.82,1228.96 \\
1229.95,1232.48,1236.97\end{array}$ & Fe VI & $4 s^{4} F-4 p^{4} D^{o}$ & $\mathrm{a}$ \\
\hline $1223.97,1240.07$ & $\mathrm{Fe} \mathrm{VI}$ & $4 s^{2} D-4 p^{2} D^{o}$ & $\mathrm{a}$ \\
\hline 1228.60, 1253.67: & Fe VI & $4 s^{2} G-4 p^{2} H^{o}$ & $\mathrm{a}$ \\
\hline 1252.79 & $\mathrm{Fe}$ VI & $4 s^{2} F-4 p^{2} G^{o}$ & $\mathrm{a}$ \\
\hline $1370.73,1374.63$ & Fe VI & $4 s^{2} G-4 p^{2} G^{o}$ & $\mathrm{a}$ \\
\hline 1371.07 & $\mathrm{Fe} \mathrm{VI}$ & $4 s^{2} F-4 p^{2} F^{o}$ & $\mathrm{a}$ \\
\hline $1011.97,1012.92,1014.84$ & $\mathrm{Fe}$ VII & $4 d^{3} F-4 f^{3} G^{o}$ & $\mathrm{a}$ \\
\hline 1052.24 & Fe VII & $4 d^{1} \mathrm{G}-4 \mathrm{f}^{1} \mathrm{H}^{\mathrm{o}}$ & $\mathrm{a}, \mathrm{b}$ \\
\hline 1068.95 & Fe VII & $4 s^{3} \mathrm{D}-4 \mathrm{p}^{1} \mathrm{~F}^{\mathrm{o}}$ & $\mathrm{a}, \mathrm{b}$ \\
\hline 1073.95 & Fe VII & $4 s^{1} \mathrm{D}-4 \mathrm{p}{ }^{1} \mathrm{P}^{o}$ & $a, b$ \\
\hline $\begin{array}{c}1080.64,1080.74,1081.21 \\
1087.86,1095.34\end{array}$ & Fe VII & $4 s^{3} D-4 p^{3} P^{o}$ & $a, b$ \\
\hline 1117.58 & Fe VII & $4 s^{1} \mathrm{D}-4 \mathrm{p}^{1} \mathrm{~F}^{\mathrm{o}}$ & $\mathrm{a}, \mathrm{b}$ \\
\hline $\begin{array}{l}1141.43,1154.99,1166.17 \\
1171.68,1173.92,1239.69\end{array}$ & Fe VII & $4 s^{3} \mathrm{D}-4 \mathrm{p}^{3} \mathrm{~F}^{\mathrm{o}}$ & $a, b$ \\
\hline 1146.45 & Fe VII & $4 s^{1} D-4 p^{3} P^{o}$ & $a, b$ \\
\hline $\begin{array}{l}1163.88,1180.82,1208.37 \\
\quad 1226.65\end{array}$ & Fe VII & $4 s^{3} D-4 p^{3} D^{0}$ & $a, b$ \\
\hline $1235.75,1244.44$ & Fe VII & $4 s^{3} D-4 p^{1} D^{o}$ & $a, b$ \\
\hline 1240.45 & Fe VII & $4 s^{1} D-4 p^{3} D^{o}$ & $a, b$ \\
\hline 1251.86 & Fe VII & $4 s^{1} D-4 p^{3} F^{o}$ & $\mathrm{a}$ \\
\hline $1006.09,1148.22$ & Fe VIII & $4 s^{2} S-3 d^{2}{ }^{2} P^{o}$ & $a, b$ \\
\hline $1062.44,1125.49$ & Fe VIII & $4 s^{2} S-4 p{ }^{2} P^{o}$ & $a, b$ \\
\hline 1075.80 & Ni VI & $4 s^{2} I-4 p^{2} H^{o}$ & $\mathrm{a}$ \\
\hline $1124.19,1144.03,1131.06$ & $\mathrm{Ni}$ VI & $4 s^{2} \mathrm{H}-4 p^{2} \mathrm{I}^{\mathrm{o}}$ & a \\
\hline 1127.93 & Ni VI & $4 s^{2} I-4 p^{2} K^{o}$ & a \\
\hline $1154.60,1161.95$ & $\mathrm{Ni}$ VI & $4 s{ }^{6} \mathrm{D}-4 \mathrm{p}^{6} \mathrm{~F}^{\mathrm{o}}$ & a \\
\hline 1163.25 & Ni VI & $4 s^{6} \mathrm{D}-4 \mathrm{p}^{6} \mathrm{P}^{o}$ & $\mathrm{a}$ \\
\hline 1176.91 & Ni VI & $4 s^{2} I-4 p^{2} I^{o}$ & $\mathrm{a}$ \\
\hline 1204.08 & Ni VI & $4 \mathrm{~s}{ }^{4} \mathrm{H}-4 \mathrm{p}{ }^{4} \mathrm{H}^{\mathrm{o}}$ & $\mathrm{a}$ \\
\hline
\end{tabular}

Table 4. Number of non-LTE levels and lines of model ions used for line formation calculations of metals.

\begin{tabular}{cccccc}
\hline \hline & III & IV & V & VI & VII \\
\hline $\mathrm{C}$ & 6,4 & 54,279 & & & \\
$\mathrm{~N}$ & 1,0 & 76,405 & 54,297 & & \\
$\mathrm{O}$ & & 83,637 & 105,671 & 54,280 & \\
$\mathrm{Ne}$ & & & 14,18 & 14,30 & 15,27 \\
$\mathrm{Si}$ & & 30,102 & 25,59 & 45,193 & 61,138 \\
$\mathrm{P}$ & 3,0 & 21,9 & 18,12 & & \\
$\mathrm{~S}$ & & & 39,107 & 25,48 & 38,120 \\
$\mathrm{Ar}$ & & & 4,0 & 48,225 & 40,130 \\
\hline
\end{tabular}

Notes. First and second number of each table entry denote the number of levels and lines, respectively. Not listed for each element is the highest considered ionization stage, which comprises its ground state only. See the text for the treatment of iron and nickel.

affects the $T_{\text {eff }}$ determination. Decreasing $\log g$ to 6.5 reduces $T_{\text {eff }}$ by about $5000 \mathrm{~K}$ and vice versa. In Fig. 5 we compare our final model for PG 1034+001 with an optical high resolution $(0.1 \AA)$ spectrum taken at the Very Large Telescope of the European Southern Observatory (ESO/VLT) within the SPY

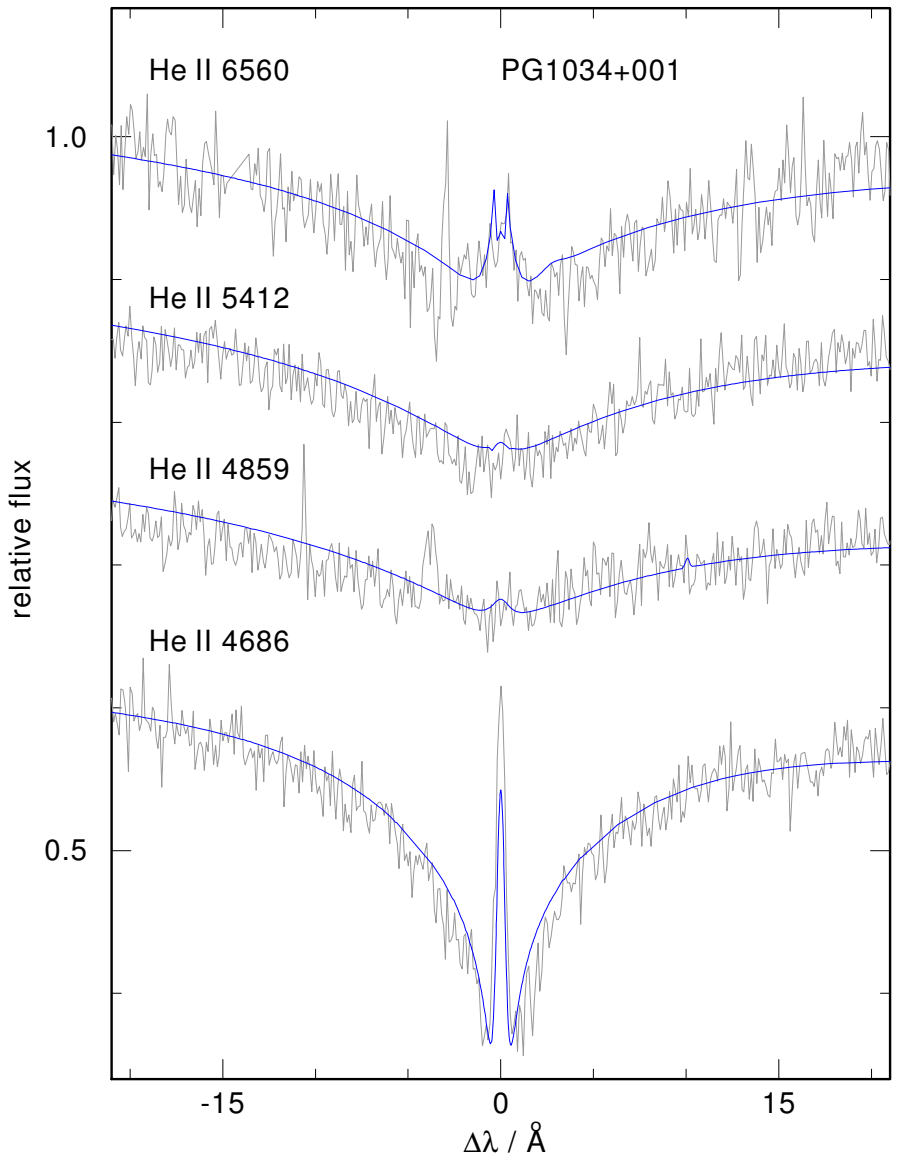

Fig. 5. Optical He II lines of PG 1034+001 compared to our final model.

survey (Napiwotzki et al. 2003). The fits to the He II lines are good although the peak height of the central emission core in the $\lambda 4686 \AA$ line is not quite matched by the model.

Hydrogen. As mentioned, $\mathrm{H}=0.05$ was determined by Dreizler et al. (1997) from a weak $\mathrm{H} \alpha$ emission core near the adjacent, stronger He II emission core in a high-resolution Keck spectrum. We used the same spectrum and found with our new models a lower value of $\mathrm{H}=0.02$ as judged from the relative strengths of the $\mathrm{H} \alpha$ and He II peak emission. From the absence of such a $\mathrm{H} \alpha$ emission near the He II emission core in PG 1034+001, we confirm the upper limit $\mathrm{H}<0.012$ determined previously (Werner 1996). Here we utilized the mentioned high-resolution SPY spectrum.

Carbon. PG 1034+001 exhibits a few CIV lines in the FUSE spectrum and the resonance doublet in the HST spectrum. The star is too hot to show the C III multiplet at $1175 \AA$. PG 0038+199 shows no C IV lines in the FUSE spectrum. Its C abundance clearly must be lower and only an upper limit can be derived. We find $\mathrm{C}=2.3 \times 10^{-4}$ and $\mathrm{C}<1.0 \times 10^{-4}$ for PG 1034+001 and PG0038+199. Both values are significantly subsolar.

Nitrogen. Both stars exhibit several prominent N IV and N V lines. It turned out that nitrogen is the most abundant metal and we found similar values, $\mathrm{N}=7.0 \times 10^{-3}$ and $1.0 \times 10^{-2}$, for 
PG 1034+001 and PG0038+199 respectively. Both values are significantly oversolar.

Oxygen. Both stars show the O VI $\lambda \lambda 1032 / 1038 \AA$ resonance doublet. PG0038+199 is hotter and additionally shows weak O VI lines at $1122 / 1125 \AA$. O V $\lambda 1371 \AA$ is present in PG 1034+001. Because of the high temperature, no other $\mathrm{O} \mathrm{v}$ line is unambiguously detected in both stars. The derived abundances are $\mathrm{O}=1.0 \times 10^{-5}$ and $1.0 \times 10^{-4}$ for PG $1034+001$ and PG 0038+199 respectively.

In the respective model for PG $1034+001$, we note that the O v $\lambda 1371 \AA$ line is too deep while the $\mathrm{O}$ VI $\lambda \lambda 1032 / 1038 \AA$ resonance doublet is too weak. Increasing $T_{\text {eff }}$ by $5000 \mathrm{~K}$ (our estimated error) only diminishes this discrepancy. A reduced abundance of $\mathrm{O}=3.2 \times 10^{-6}$ would fit the O v $\lambda 1371 \AA$ line but, of course, the O VI doublet fits even worse. An interstellar contribution to the doublet cannot be excluded and might explain the too-weak model doublet. In any case, the derived oxygen abundances are significantly subsolar.

Neon. The Ne VII $\lambda 973.33 \AA$ line is the only means to access the neon abundance. Unfortunately, the line is blended with an interstellar $\mathrm{H}_{2}$ line in both stars. We are therefore only able to derive an upper limit, which is about solar for both stars.

Silicon. A number of Si IV and Siv lines are detectable in both stars. Because of the higher temperature, the Si v lines are more pronounced in PG $0038+199$. We derive almost solar abundances: $\mathrm{Si}=5.0 \times 10^{-4}$ and $1.0 \times 10^{-3}$ for PG $1034+001$ and PG 0038+199.

Phosphorus. The $\mathrm{PV}$ resonance doublet was used to measure the $\mathrm{P}$ abundance. The short-wavelength component at $1117.98 \AA$ is located within a blend of two other photospheric lines (S VI and Fe VII). We find roughly solar abundances: $\mathrm{P}=2.9 \times 10^{-6}$ and $1.0 \times 10^{-5}$ for PG $1034+001$ and PG 0038+199.

Sulfur. We used the S VI resonance lines as well as the S VI line at $1117.76 \AA$ within the blend mentioned above to determine the abundances $S=5.0 \times 10^{-5}$ and $1.0 \times 10^{-4}$ for PG $1034+001$ and PG 0038+199, which are close to the solar value.

Argon. Only one argon line is available for an abundance determination, namely, Ar VII $\lambda 1063.55 \AA$. In PG 0038+199, however, it is not detectable because of a strong interstellar $\mathrm{H}_{2}$ line blend. The argon line is seen in PG 1034+001 and a weak $\mathrm{H}_{2}$ line blend is located in its red wing. We derive $\mathrm{Ar}=1.0 \times 10^{-4}$, which is close to solar. At this abundance, the computed line is not quite as strong as the observed one, however, a higher abundance can be excluded because our models then predict other Ar VII lines that are not observed. The $f$-value of this line in several databases differs by about $20 \%$ according to Werner et al. (2007), but this cannot explain the discrepancy because a ten times higher $f$-value would be necessary to fit the observation. With a test calculation, we have checked that extending the argon model atom by Ar IX and including a more detailed Ar VIII model ion does not solve the problem.

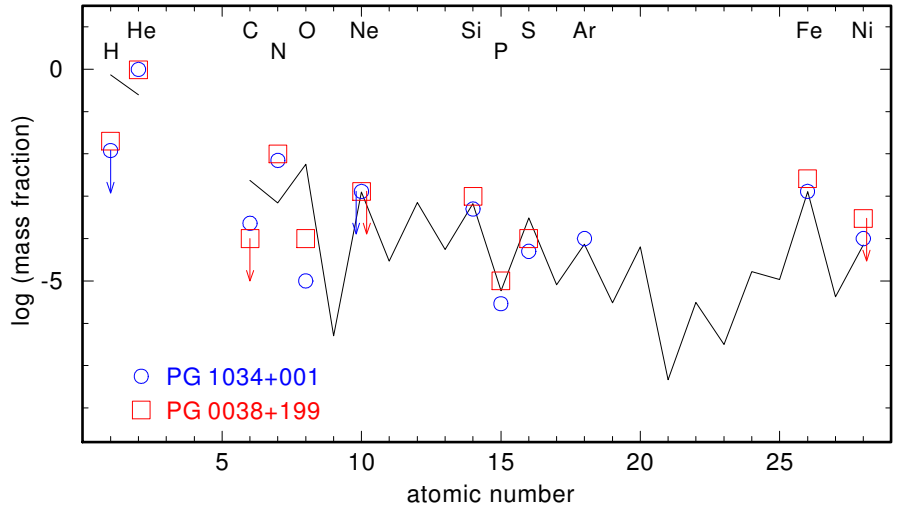

Fig. 6. Element abundances measured in PG 1034+001 and PG 0038+199. Upper limits are indicated by arrows. The black solid line indicates solar abundances.

Iron. Both stars exhibit prominent lines from Fe VII. In addition, we see Fe VIII lines that are stronger in PG 0038+199 than in PG 1034+001 because of its higher temperature. For the same reason, Fe VI lines are seen only in the cooler star PG 1034+001. Within error limits, the derived iron abundance is solar: $\mathrm{Fe}=$ $1.3 \times 10^{-3}$ and $2.6 \times 10^{-3}$ for PG $1034+001$ and PG 0038+199.

Nickel. Some lines of Ni VI can be detected in PG 1034+001, and we derive a nickel abundance that is close to solar $(\mathrm{Ni}=$ $\left.1.0 \times 10^{-4}\right)$. At the higher temperature of PG $0038+199$, these lines are too weak to be seen at solar abundance. We derive an upper limit of $\mathrm{Ni}<3.0 \times 10^{-4}$.

The error for the abundance determinations is $\pm 0.3 \mathrm{dex}$ as estimated from models with effective temperature and gravity at the respective error limits. In view of the difficulties in fitting the oxygen lines in PG 1034+001, a larger error of 0.5 dex for the $\mathrm{O}$ abundance is estimated. Because of the very strong temperature dependence of the only available neon line, an error of one dex for neon is realistic. The abundances are summarized in Table 1 and displayed in Fig. 6.

\section{Summary and discussion}

From UV spectroscopy we have re-determined $T_{\text {eff }}$ and $\log g$ of two of the hottest known DO white dwarfs. In particular, the error in $T_{\text {eff }}$ could be strongly reduced, which is the basis of precise abundance determinations. We measured the abundances (or upper limits) of hydrogen and several metals (Table 1).

While most of the lines observed in these objects are matched by our models, they do not reproduce the oxygen and argon lines in PG 1034+001. In the model with an $\mathrm{O}$ abundance that fits the O V $\lambda 1371 \AA$ line, the OVI $\lambda \lambda 1032 / 1038 \AA$ resonance doublet is too weak. An ISM contribution to the observed O VI doublet could explain this discrepancy. At the adopted argon abundance, the only observed line of this species, Ar VII $\lambda 1063.55 \AA$, is too weak in the model. Increasing the Ar abundance results in other lines that are not observed. The $f$-value of the $\lambda 1063.55 \AA$ line is well known and applying values from different databases hardly affects the computed line profile. Thus, this discrepancy remains an unresolved issue.

The main result of our study is that the heavy metal abundances $(\mathrm{Ne}, \mathrm{Si}, \mathrm{P}, \mathrm{S}, \mathrm{Ar}, \mathrm{Fe}, \mathrm{Ni}$ ) are compatible with the solar abundance pattern. We can therefore assume that radiative levitation and gravitational settling do not affect the chemical composition of the photospheres and, hence, the abundances 
K. Werner et al.: FUV spectroscopy of two extremely hot, helium-rich white dwarfs

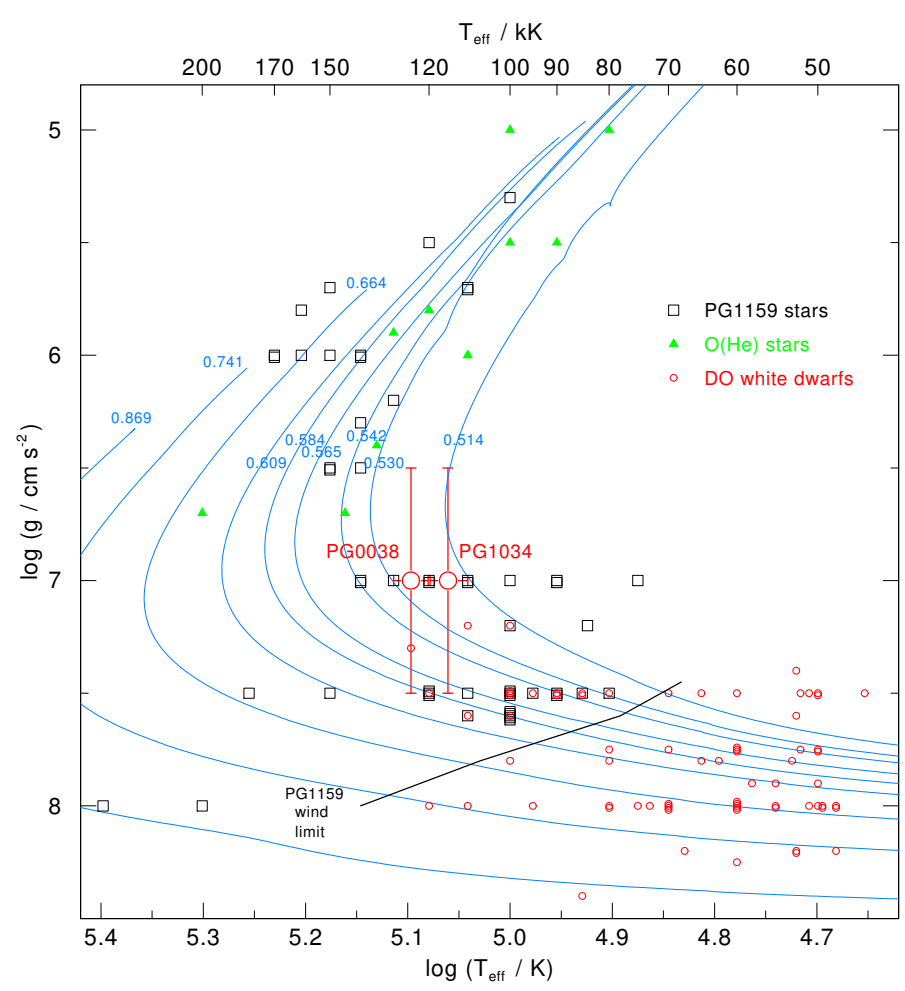

Fig. 7. Location of PG $1034+001$ and PG $0038+199$ in the $g-T_{\text {eff }}$ diagram (circles with error bars) among other DO white dwarfs, PG 1159 stars, and $\mathrm{O}(\mathrm{He})$ stars. Evolutionary tracks from Althaus et al. (2009) are labeled with the stellar mass in $M_{\odot}$. Indicated is the PG1159 wind limit according to Unglaub \& Bues (2000). Below it, no PG 1159 stars are found because radiation-driven stellar winds become so weak that they can no longer prevent gravitational settling of heavy elements.

of the light metals $(\mathrm{C}, \mathrm{N}, \mathrm{O})$ can be compared to helium-rich pre-white dwarfs in order to conclude on evolutionary links. Carbon and oxygen are significantly subsolar while nitrogen is oversolar. Thus, the two DO white dwarfs cannot be descendants of PG1159 stars (as suspected previously, Werner et al. 1995) which show the opposite enrichment and depletion of the CNO elements. Instead, they are obviously evolved $\mathrm{O}(\mathrm{He})$ stars which have rather similar abundances. This was already stated by Reindl et al. (2014b), although at that time it could not be excluded that diffusion had affected the two DOs' photospheric abundances.

The positions of the two DOs investigated here among other hydrogen-deficient objects in the $g-T_{\text {eff }}$ diagram are displayed in Fig. 7. They may be regarded as immediate successors of the $\mathrm{O}(\mathrm{He})$ stars and predecessors of more evolved DO white dwarfs in which gravitational settling and (at still high enough effective temperature) radiative levitation determine the heavy element abundances. Reindl et al. (2014b) conclude that the CNO abundances of $\mathrm{O}(\mathrm{He})$ stars cannot be the result of single-star evolution but can be better explained as the outcome of closebinary evolution. Through the detailed $\mathrm{CNO}$ abundance pattern as well as the identification of residual hydrogen (as is the case in PG 0038+199), different varieties of binary WD merger or common-envelope evolutionary sequences may be invoked. Another unsolved problem in the context of evolutionary considerations is the presence of planetary nebulae $(\mathrm{PNe})$ around some $\mathrm{O}(\mathrm{He})$ stars, which is also relevant here because one of our DOs
(PG 1034+001) has a PN (although its true nature as a PN is debated, Hewett et al. 2003; Rauch et al. 2004; Chu et al. 2004). For a thorough discussion of these topics we refer to Reindl et al. (2014b).

Acknowledgements. We thank Ralf Napiwotzki for putting the reduced ESO/VLT spectrum of PG 1034+001 at our disposal. T. Rauch was supported by the German Aerospace Center (DLR) under grant 50 OR 1507. The TMAD service (http://astro-uni-tuebingen.de/ TMAD) used to compile atomic data for this paper was constructed as part of the activities of the German Astrophysical Virtual Observatory. This research has made use of the SIMBAD database, operated at CDS, Strasbourg, France, and of NASA's Astrophysics Data System Bibliographic Services. Some of the data presented in this pape were obtained from the Mikulski Archive for Space Telescopes (MAST). This work had been done using the profile fitting procedure OWENS, developed by M. Lemoine and the FUSE French Team.

\section{References}

Althaus, L. G., Panei, J. A., Miller Bertolami, M. M., et al. 2009, ApJ, 704, 1605 Anderson, L. S. 1989, ApJ, 339, 558

Asplund, M., Grevesse, N., Sauval, A. J., \& Scott, P. 2009, ARA\&A, 47, 481

Bashkin, S. \& Stoner, J. O. 1975, Atomic energy levels and Grotrian Diagrams, Vol. 1, Hydrogen I - Phosphorus XV) Vol. 2: Sulfur I - Titanium XXII (Amsterdam: North-Holland Publ. Com)

Chu, Y.-H., Gruendl, R. A., Williams, R. M., Gull, T. R., \& Werner, K. 2004, AJ, 128,2357

Dere, K. P., Landi, E., Mason, H. E., Monsignori Fossi, B. C., \& Young, P. R. 1997, A\&AS, 125, 149

Dreizler, S., \& Werner, K. 1996, A\&A, 314, 217

Dreizler, S., Werner, K., Heber, U., Reid, N., \& Hagen, H. 1997, in The 3d Conference on Faint Blue Stars, eds. A. G. D. Philip, J. Liebert, R. Saffer, \& D. S. Hayes, 303

Green, R. F., Schmidt, M., \& Liebert, J. 1986, ApJS, 61, 305

Hébrard, G., \& Moos, H. W. 2003, ApJ, 599, 297

Hébrard, G., Friedman, S. D., Kruk, J. W., et al. 2002, Planet. Space Sci., 50, 1169

Hewett, P. C., Irwin, M. J., Skillman, E. D., et al. 2003, ApJ, 599, L37

Kurucz, R. L. 1991, in Stellar Atmospheres - Beyond Classical Models, eds. L. Crivellari, I. Hubeny, \& D. G. Hummer, NATO ASIC Proc., 341, 441

Kurucz, R. L. 2009, in AIP Conf. Ser., eds. I. Hubeny, J. M. Stone, K. MacGregor, \& K. Werner, 1171, 43

Kurucz, R. L. 2011, Can. J. Phys., 89, 417

Landi, E., Young, P. R., Dere, K. P., Del Zanna, G., \& Mason, H. E. 2013, ApJ, 763,86

Mahsereci, M. 2011, Diploma Thesis, Eberhard Karls Universität Tübingen

Mahsereci, M., Ringat, E., Rauch, T., Werner, K., \& Kruk, J. W. 2012, in IAU Symp., 283, 426

Napiwotzki, R., Christlieb, N., Drechsel, H., et al. 2003, The Messenger, 112, 25 Rauch, T., \& Deetjen, J. L. 2003, in Stellar Atmosphere Modeling, eds. I. Hubeny, D. Mihalas, \& K. Werner, ASP Conf. Ser., 288, 103

Rauch, T., Kerber, F., \& Pauli, E.-M. 2004, A\&A, 417, 647

Reindl, N., Rauch, T., Werner, K., et al. 2014a, A\&A, 572, A117

Reindl, N., Rauch, T., Werner, K., Kruk, J. W., \& Todt, H. 2014b, A\&A, 566, A116

Seaton, M. J., Yan, Y., Mihalas, D., \& Pradhan, A. K. 1994, MNRAS, 266, 805

Unglaub, K., \& Bues, I. 2000, A\&A, 359, 1042

Werner, K. 1996, A\&A, 309, 861

Werner, K., \& Dreizler, S. 1999, J. Comput. Appl. Math., 109, 65

Werner, K., \& Herwig, F. 2006, PASP, 118, 183

Werner, K., \& Rauch, T. 2015, A\&A, 583, A131

Werner, K., Dreizler, S., \& Wolff, B. 1995, A\&A, 298, 567

Werner, K., Rauch, T., Kruk, J. W., \& Kurucz, R. L. 2011, A\&A, 531, A146

Werner, K., Deetjen, J. L., Dreizler, S., et al. 2003, in Stellar Atmosphere Modeling, eds. I. Hubeny, D. Mihalas, \& K. Werner, ASP Conf. Ser., 288, 31

Werner, K., Rauch, T., \& Kruk, J. W. 2007, A\&A, 466, 317

Werner, K., Dreizler, S., \& Rauch, T. 2012, TMAP: Tübingen NLTE ModelAtmosphere Package, Astrophysics Source Code Library

[record ascl: 1212.015]

Werner, K., Rauch, T., \& Kepler, S. O. 2014, A\&A, 564, A53

Williger, G. M., Oliveira, C., Hébrard, G., et al. 2005, ApJ, 625, 210 